\section{Reverse Takotsubo syndrome, a case report of a rare cause for postpartum heart failure}

\author{
Leo Kilian, ${ }^{1}$ Philip Haaf, ${ }^{2}$ \\ Otmar Pfister, ${ }^{2}$ Annina S. Vischer, ${ }^{1}$ \\ Olav Lapaire, ${ }^{3}$ Thilo Burkard ${ }^{1,2}$ \\ ${ }^{1}$ Medical Outpatient and Hypertension \\ Clinic, ESH Hypertension Centre of \\ Excellence; ${ }^{2}$ Department of Cardiology; \\ ${ }^{3}$ Department of Gynecology and \\ Pregnancy Medicine, University \\ Hospital Basel, Basel, Switzerland
}

\section{Abstract}

Predominant causes for newly diagnosed postpartum heart failure are preeclampsia and peripartum cardiomyopathy. Being an anatomical variant of Takotsubo syndrome (TTS) reverse TTS in this period is rare. We present a 36 year old patient, who had delivered triplets by cesarean section. Because of postpartum bleeding she was administered sulprostone. Later she was transferred to the Intensive Care Unit with sudden development of dyspnea, tachypnea and tachycardia. Clinical symptoms, laboratory findings and chest radiograph showed signs of acute heart failure. Transthoracic echocardiography (TTE) revealed reverse TTS with moderately reduced left ventricular ejection fraction (LVEF 39\%). The patient stabilized with loop diuretic, angiotensine-converting enzyme inhibitors and beta-blockade. Breast-feeding was discouraged and bromocriptine administered. Left ventricular function normalized (LVEF $60 \%$ ) within four weeks. TTS should be considered in patients with early postpartum development of heart failure. Rapid cardiac recompensation after the start of adequate therapy and complete resolution of clinical symptoms and TTE findings are typical for postpartum TTS.

\section{Introduction}

Heart failure during and after pregnancy can be caused by e.g. preexisting cardiovascular disease, hypertensive complications (e.g. preeclampsia, HELLPsyndrome), pulmonary embolism, peripartum cardiomyopathy (PPCM), previously unrecognized genetic forms of cardiomyopathies, myocarditis. ${ }^{1}$ Pregnancy related takotsubo syndrome (TTS) as cause of acute heart failure has been described in rare cases, with e.g. 18 cases identified by Minatoguchi et al. in 2014. ${ }^{2}$ We present a case of a rare variant of postpartum TTS, with a hyperkinetic cardiac apex and wall motion abnormalities at the base of the left ventricle, also known as Reverse TTS. Only few cases with this rare anatomical variant of TTS related to pregnancy have been described. ${ }^{3-8}$

\section{Patient information and physical examination}

We present a 36 year old patient, who delivered triplets by cesarean section at week $32+5$ of pregnancy (secundigravida, 1 missed abortion) (Table 1). During cesarean section a Bakri ${ }^{\circledR}$ Balloon had been placed to prevent postpartum hemorrhage and tranexamic acid was given. Yet because of insufficient uterus-contraction and postpartum bleeding additionally sulprostone (approximately $500 \mu \mathrm{g}$ ) was administered, followed by nasal oxytocin (4 units four times a day) for 3 days.

On the third day after cesarean section the patient developed dyspnea, tachypnea and tachycardia. Respiration rate was $20 / \mathrm{min}$, pulse oximetry showed $90 \%$ oxygenation, heart rate was 145 beats per minute and blood pressure was 133/84 mmHg. General examination was significant for a patient in distress with intermittent expiratory stridor. Physical examination showed slight peripheral pitting edema without distended jugular veins, hepatojugular reflux was negative. Auscultation of the lungs revealed bilateral basal rales, cardiac auscultation was normal.

\section{Diagnostic assessment and inter- ventions}

Electrocardiography showed sinus tachycardia with no ST-segment elevation or depression, but T-inversion in aVL and I. A chest radiograph revealed pulmonary congestion, bilateral pleural effusions and cardiomegaly. High-sensitivity cardiac Troponin T was elevated at $33 \mathrm{ng} / \mathrm{L}$ (cut-off value $14 \mathrm{ng} / \mathrm{L}$ ), Creatine Kinase-MB 3.8 $\mu \mathrm{g} / \mathrm{L}$ (cut-off value $5 \mu \mathrm{g} / \mathrm{L}$ ), D-Dimer at $1.18 \mu \mathrm{g} / \mathrm{mL}$ (cut-off value $0.5 \mu \mathrm{g} / \mathrm{mL}$ ) and NT-proBNP at $9254 \mathrm{ng} / \mathrm{L}$ (cut-off value 177 $\mathrm{ng} / \mathrm{L}$ ), hemoglobin was $82 \mathrm{~g} / \mathrm{L}$ (lower cutoff value $100 \mathrm{~g} / \mathrm{L}$ ). CT-angiography of the chest showed no signs of pulmonary embolism. A transthoracic echocardiography (TTE) showed a moderately reduced left ventricular ejection
Correspondence: Thilo Burkard, Department of Cardiology, University Hospital Basel Petersgraben 4, 4031 Basel, Switzerland.

Tel.: +41.613287738.

E-mail: thilo.burkard@usb.ch

Key words: Takotsubo; peripartum cardiomyopathy; sulprostone; case report; bromocriptin; postpartum heart failure.

Contributions: LK, AV, TB, OP, PH, were involved in compilation of data and writing of this manuscript; TB, OL, PH were involved in the management of the case.

Consent: the author/s confirm that written consent for submission and publication of this case report including image(s) and associated text has been obtained from the patient.

Conflict of interest: the authors declare no potential conflict of interest.

Received for publication: 3 July 2018

Revision received: 7 September 2018.

Accepted for publication: 8 September 2018 .

This work is licensed under a Creative Commons Attribution NonCommercial 4.0 License (CC BY-NC 4.0).

(C) Copyright L. Kilian et al., 2018

Licensee PAGEPress, Italy

Cardiogenetics 2018; 8:7671

doi:10.4081/cardiogenetics.2018.7671

fraction (LVEF) of $39 \%$. Contractility of the apex was hyper dynamic with a hypo- to akinesia of all basal segments (link to sequence for online publication). A small pericardial effusion was seen near the right atrium. We presumed postpartum heart failure due to reverse TTS with the differential diagnosis of PPCM. Two days later cardiac magnetic resonance imaging (Figure 1) confirmed an apical hyperkinesia with basal to midventricular hypokinesia and akinesia without evidence of myocardial edema in T2 mapping sequences. Bilateral pleural effusions were still present. A myocardial edema in the areas of wall motion abnormality was not found. There was no evidence of myocardial late gadolinium enhancement (Figure 2). By the cardiac MRI, we were able to exclude myocardial infarction or myocarditis as the cause of her acute heart failure. ${ }^{9}$

The initial therapy for acute heart failure was intravenous loop diuretic (furosemide $40 \mathrm{mg}$ ) and $30 \mathrm{mg}$ of labetalol because of tachycardia. Another $40 \mathrm{mg}$ of furosemide were administered when clinical benefit from the first dose was insufficient, which then led to a diuresis of 
$2 \mathrm{~L}$ over $2 \mathrm{~h}$. Patient received non-invasive ventilation and was transferred to the Intensive Care Unit (ICU). Treatment was adapted with a short acting beta-blocker (propanolol $10 \mathrm{mg}$ three times daily), ACEI (enalapril $5 \mathrm{mg}$ ) and intravenous loopdiuretic (furosemide). Because the patient wished to stop breast-feeding and the initial differential diagnosis of PPCM bromocriptine $2.5 \mathrm{mg}$ twice a day was started according to current recommendations. ${ }^{10}$ Beta-blockade was changed to carvedilol (6.5 mg/day) and we interchanged enalapril to ramipril (5 $\mathrm{mg}$ /day). Receiving a total of $120 \mathrm{mg}$ furosemide the resulting diuresis of $5 \mathrm{~L}$ in the first $24 \mathrm{~h}$ led to complete clinical stabilization and decrease of NT-proBNP to 7904 ng/L.

During her stay on ICU, clinical situation improved, ACE-I and betablockade could be increased, loop-diuretics were decreased and changed to an oral form (torasemide). Patient could be transferred to regular ward after 3 days.

\section{Follow-up and outcomes}

Before hospital discharge 14 days after admission to the ICU there were no signs of heart failure in the physical examination anymore. Accordingly, no pleural effusions were found with ultrasound anymore. The patient was discharged from the hospital.
4 weeks after discharge, the global and regional left ventricular function was normalized in TTE. LVEF was measured at $60 \%$. Accordingly, NT-proBNP reached normal level ( $85 \mathrm{ng} / \mathrm{L})$.

Over the next months the ACEInhibition and beta-blockade was tapered due to low blood pressure and orthostatic hypotension and stopped completely after 7 months. TTE and NT-proBNP still remained normal after 9 months.

\section{Discussion and Conclusions}

In the presented case, the patient showed a sudden development of symptoms of postpartum heart failure three days after delivery of triplets by cesarean section. TTE findings were leading us to the diagnosis of reverse TTS.

For diagnosis we followed the revised Mayo-criteria of the Takotsubo Italian Network and Heart Failure Association of the European Society of Cardiology diagnostic criteria for Takotsubo syndrome. ${ }^{11,12}$ Following these criteria, we did not exclude an acute coronary plaque rupture, dissection, thrombosis or spasm by coronary angiography. Yet we found Tinversion in ECG without ST-segment elevation or depression supporting our diagnosis. In the laboratory results we saw a significant and completely reversible elevation of NT-proBNP. Creatine-Kinase-
MB never exceeded the above mentioned $3.8 \mu \mathrm{g} / \mathrm{L}$ and high-sensitivity Troponin $\mathrm{T}$ never exceeded $33 \mathrm{ng} / \mathrm{L}$, also supporting the diagnosis of TTS. Yet it is possible that the increase seen for Troponin could be explained by the postpartum state alone. Late gadolinium enhancement in the MRI was negative, which is considered typical for TTS, further strengthening our diagnosis by imaging and also excluding myocarditis. ${ }^{13}$ But the MRI missed proof of myocardial edema in the areas of wall motion abnormality as is common in TTS. Under therapy with beta-blockade, ACE-I and loop diuretics we achieved a rapid recovery during $48 \mathrm{~h}$ and complete recovery of left ventricular function after 4 weeks, as confirmed in TTE. Cesarean section and birth were viable stressful events preceding the development of the first symptoms.

Considering other possible diagnoses for this patient there were no signs of myocarditis. A genetic form of a cardiomyopathy was improbable, as a complete recovery was seen after 9 months. Also there were no findings for preeclampsia. Acute heart failure due to peripartum cardiomyopathy (PPCM) would differ in the evolution of the disease and left ventricular dysfunction, as well as NTproBNP would not normalize within four weeks. ${ }^{1}$ A systematic review by Citro et al. led to the conclusion that peripartum TTS should be considered a clinical entity different from PPCM. ${ }^{14}$ A sudden onset of symptoms with a rapid worsening of the

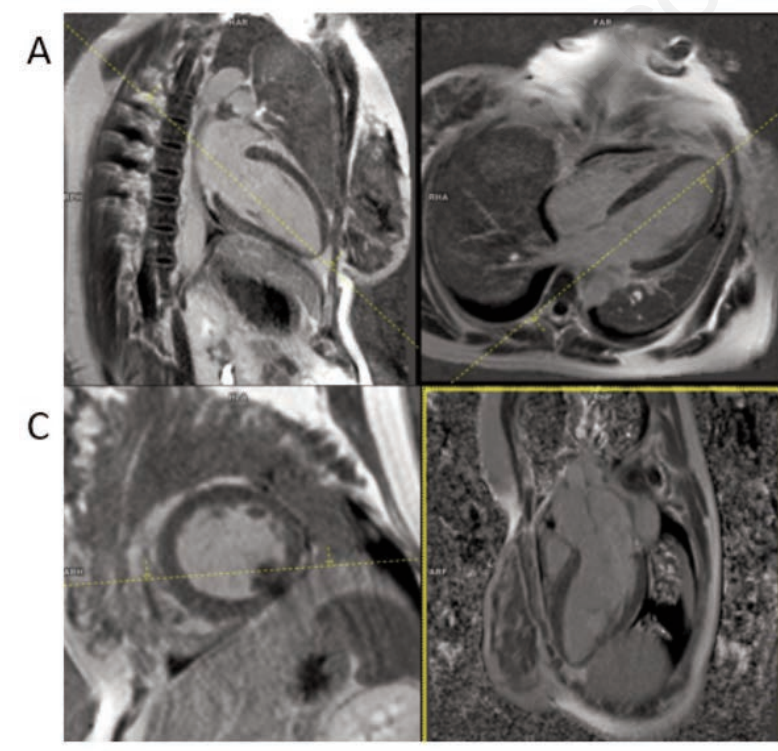

B

Figure 1. Late gadolinium enhancement showed no contrast uptake of the myocardium and bilateral pleural effusion (right $>$ left). A) 2 chamber long axis; B) 4-chamber long axis; C) short axis at the level of the papillary muscles; D) 3-chamber long axis

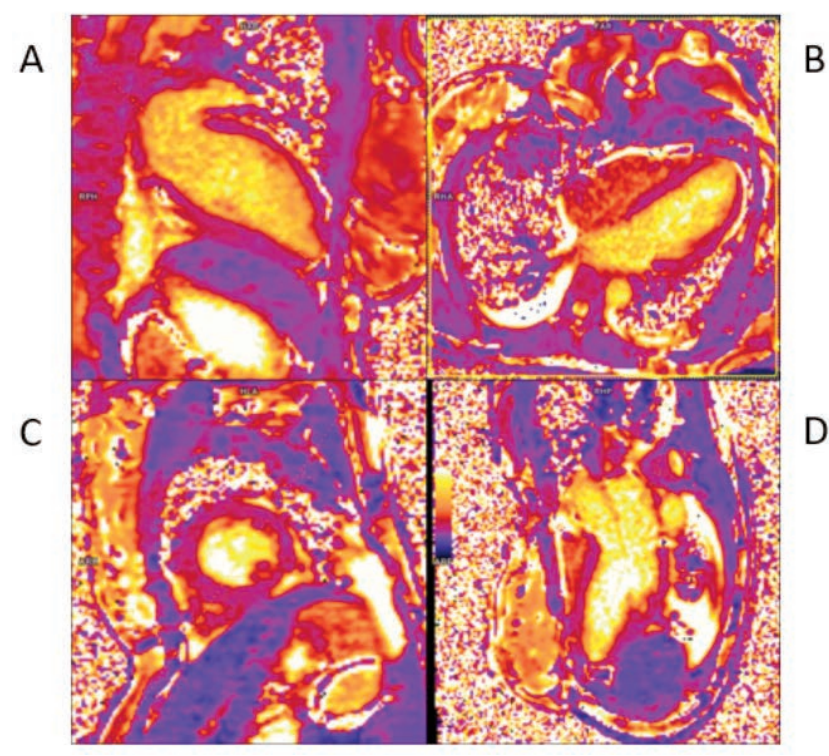

Figure 2. T2 mapping showed no evidence of myocardial edema and bilateral pleural effusion (right>left). A) 2-chamber long axis; B) 4-chamber long axis; C) short axis at the level of the papillary muscles; D) 3-chamber long axis. 
clinical presentation requiring immediate therapy is considered typical for TTS. In contrast to TTS a complete normalization of left ventricular function was only observed in a minority of patients with PPCM and clinical symptoms were increasing over time. ${ }^{12}$ Physical and emotional stress, as wells as administration of catecholamines/ vasoconstrictive substances during cesarean section have been discussed to cause pregnancy related TTS, with the majority of cases developing symptoms at time of cesarean section up to 6 days later. ${ }^{2,12}$ The main risk factor for development of TTS in the postpartum period seems to be the cesarean section itself, which often comprises stress, emotionally and physically, as well as the administration of adrenergic stimulants and anticholinergics. $^{12,15}$ In our case the administration of sulprostone after the cesarean section can be discussed as an additional cause for the development of TTS, as sulprostone has been associated with TTS before. ${ }^{16,17}$ Diffuse coronary spasm alone due to the sulprostoneadministration as a theoretical cause for TTS is possible. Still, the administration was stopped before the patient developed the first clinical symptoms of acute heart failure.

We did not exclude a pheochromocytoma as requested in above mentioned diagnostic criteria.

Following the above mentioned criteria we miss proof for the absence of acute coronary plaque rupture, dissection, thrombosis or spasm as coronary angiography was not done. The clinical and diagnostic follow up nevertheless showed no signs for a coronary heart disease. We also miss myocardial edema in the areas of wall motion abnormality in the MRI, as it is present in approx. $80 \%$ of TTS cases. ${ }^{18}$ Yet we saw no late gadolinium enhancement and no signs for myocarditis or myocardial infarction. MRI was performed when the patient had already stabilized, while TTE was performed while she was still in acute heart failure. The remaining criteria were fulfilled following either the revised Mayocriteria of the Takotsubo Italian Network or the Heart Failure Association of the European Society of Cardiology diagnostic criteria for Takotsubo syndrome. ${ }^{11,12}$

Meanwhile new international criteria the InterTAK Diagnostic Criteria and Diagnostic Score/Algorithm were published (Tables 2 and 3). ${ }^{19,20}$ Following these new criteria, pheochromocytoma no longer have to be excluded as the findings during a catecholamine storm are considered indistinguishable from TTS. ${ }^{19,20}$ With the new InterTAK Diagnostic Score our patient scores 74 points (Female Sex 25 points, Emotional Stress 24 points, Physical Stress 13 points, No ST-segment depression 12 points), which gives a high probability for TTS (cut off 70 points). The TTE and MRI findings as well as the clinical development we saw in our patient further support our diagnosis following these new diagnostic score and algorithm. Yet again we miss the exclusion of coronary artery disease with coronary computed tomography

Table 1. Timeline.

\begin{tabular}{|c|c|}
\hline 4 weeks before admission ICU & Admission to hospital because of shortened cervical length during pregnancy with dichorial-triamniotic triplets \\
\hline 3 days before admission to ICU & $\begin{array}{l}\text { Caesarean section, with Bakri Balloon placement and due to atone uterus with postpartum bleeding administration } \\
\text { of sulprostone followed by oxytocin }\end{array}$ \\
\hline At admission to ICU & $\begin{array}{l}\text { Acute heart failure presenting with dyspnea, tachycardia, pleural effusions, slight peripheral edema, NT-proBNP } \\
9254 \text { ng/L } \\
\text { TTE showed wall motion abnormalities, LVEF } 39 \% \\
\text { Loop-diuretic, beta-blockade and ACE-inhibition were started }\end{array}$ \\
\hline 2 days after admission to ICU & $\begin{array}{l}\text { MRI without typical edema } \\
\text { Patient stabilized, NT pro BNP at } 6421 \mathrm{ng} / \mathrm{L} \\
\text { Bromocriptine was started, patient stopped breast feeding }\end{array}$ \\
\hline 14 days after admission to ICU & Patient still stable, left hospital \\
\hline 4 weeks after presentation & $\begin{array}{l}\text { TTE showed no regional wall motion abnormalities with LVEF } 60 \% \text { and NT-proBNP } 85 \mathrm{ng} / \text {. } \\
\text { No pleural effusions or other signs of heart failure } \\
\text { Therapy: Beta-blockade and ACE-Inhibition }\end{array}$ \\
\hline 9 months after presentation & Medication was stopped, TTE and NT pro BNP remained normal \\
\hline
\end{tabular}

\section{Table 2. International Takotsubo Diagnostic Criteria (InterTAK Diagnostic Criteria). ${ }^{19}$}

1. Patients show transient* left ventricular dysfunction (hypokinesia, akinesia or dyskinesia) presenting as apical ballooning or midventricular, basal or focal wall motion abnormalities. Right ventricular involvement can be present. Besides these regional wall motion patterns, transitions between all types can exist. The regional wall motion abnormality is present in the subtended myocardial territory of a single coronary artery (focal TTS) ${ }^{\circ}$

2. An emotional, physical or combined trigger can precede the takotsubo syndrome event, bit this is not obligatory

3. Neurologic disorders (e.g. subarachnoidal haemorrhage, stroke/transient ischaemic attack or seizures) as well as pheochromocytoma may serve as triggers for takotsubo syndrome

4. New ECG abnormalities are present (ST-segment elevation, ST-segment depression, T-wave inversion and QTc prolongation); however, rare cases exist without any ECG changes

5. Levels of cardiac biomarkers (troponin and creatine kinase) are moderately elevated in most cases; significant elevation of brain natriuretic peptide is common

6. Significant coronary artery disease is not a contradiction in takotsubo syndrome

7. Patients have no evidence of infectious myocarditis ${ }^{\circ}$

8. Postmenopausal women are predominantly affected

*Wall motion abnormalities may remain for al prolonged period of time or documentation of recovery may not be possible. For example, death, before evidence of recovery is captured; ${ }^{\circ}$ cardiac magnetic resonance imaging is recommended to exclude infectious myocarditis and diagnosis confirmation of Takotsubo syndrome. 
Table 3. InterTAK Diagnostic Score. ${ }^{20}$

\begin{tabular}{ll}
\hline Female sex & 25 points \\
Emotional stress & 24 points \\
\hline Physical stress & 13 points \\
No ST-segment depression & 12 points \\
\hline Psychiatric disorders & 11 points \\
Neurologic disorders & 9 points \\
QTc prolongation & 6 points \\
$<=70$ points & $>70$ points \\
\hline Low/intermediate probability of TTS & High probability of TTS \\
\hline
\end{tabular}

angiography, as it is recommended in this algorithm.

\section{Online videos}

The sequence of online videos shows cine loops of the first echocardiography performed in the intensive care unit showing hyperkinetic apex and wall motion abnormalities in the basal segments: Video 1) 4-chamber view; Video 2) 2-chamber view; Video 3) 3-chamber view.

\section{References}

1. Hilfiker-Kleiner D, Haghikia A, Nonhoff J, Bauersachs J. Peripartum cardiomyopathy: current management and future perspectives. Eur Heart J 2015;36:1090-7.

2. Minatoguchi M, Itakura A, Takagi E, et al. Takotsubo cardiomyopathy after cesarean: A case report and published work review of pregnancy-related cases. J Obstet Gynaecol Res 2014;40: 1534-9.

3. Ledakowicz-Polak A, Bartodziej J, Majos A, Zielińska M. Inverted stressinduced cardiomyopathy as a unusual variant of acute heart failure after cesarean delivery - a case report. BMC Cardiovas Disor 2016;16:76.

4. Lee S, Lee KJ, Yoon HS, et al. Atypical Transient Stress-Induced Cardiomyopathies with an Inverted
Takotsubo Pattern in Sepsis and in the Postpartal State. Tex Heart I J 2010;37:88-91

5. Belliveau D, Sabe D. Reverse Takotsubo Cardiomyopathy following Exogenous Epinephrine Administration in the Early Postpartum Period. Echocardiogr J Card 2016;33:1089-91.

6. Ashrafpoor G, Puymirat E, Sabbah L, et al. Inverted stress (Takotsubo) cardiomyopathy following Caesarean section: Insights from cardiac magnetic resonance. Int J Cardiol 2013;102:38-9.

7. Gleich S, Barbara D, Arendt K, et al. Reverse Apical Ballooning Echocardiographic Pattern in Eclampsia-Related Cardiomyopathy. Case Rep 2016;6:6-9.

8. Papanikolaou J, Makris D, Tsolaki V, et al. Post-partum hemorrhage complicated by reverse-Takotsubo cardiogenic shock; a novel therapeutic approach. Am J Emerg Med 2017;35:935-6.

9. Patel N, Kolakalapudi P, Arora G. Contrast application in cardiac magnetic resonance. Echocardiogr J Card 2018;35:401-9.

10. Hilfiker-Kleiner D, Haghikia A, Berliner D, et al. Bromocriptine for the treatment of peripartum cardiomyopathy: a multicentre randomized study. Eur Heart J 2017;38:2671-9.

11. Parodi G, Citro R, Bellandi B, et al. Revised clinical diagnostic criteria for Tako-tsubo syndrome: The Tako-tsubo Italian Network proposal. Int J Cardiol 2014;172: 282-3.

12. Lyon A, Bossone E, Schneider B, et al.
Current state of knowledge on Takotsubo syndrome: a position statement from the task force on Takotsubo syndrome of the Heart Failure Association of the European Society of Cardiology. Eur J Heart Fail 2016;18:827.

13. Kohan AA, Levy Yeyati E, De Stefano L, et al. Usefulness of MRI in takotsubo cardiomyopathy: a review of the literature. Cardiovas Diagn Ther 2014;4:13846.

14. Citro R., Giudice R, Mirra M, et al. Is Tako-tsubo syndrome in the postpartum period a clinical entity different from peripartum cardiomyopathy. Cardiovasc Med 2013;14:568-75.

15. Zdanowicz JA, Utz AC, Bernasconi I, et al. "Broken heart" after cesarean delivery. Case report and review of literature. Arch Gynecol Obstet 2011;283:687-94.

16. Guerci P, Novy E, Vital F, et al. Sulprostone for postpartum hemorrhage in a parturient with a history of Takotsubo cardiomyopathy. J Clin Anesth 2013;25:327-30

17. Courand P-Y, Jenck S, Cassar E, et al. Tako-tsubo Cardiomyopathie Probably Associated With Administration of Sulprostone Following Cesarean Delivery. Rev Esp Cardiol 2012;65:770-71.

18. Eitel I, von Knobelsdorff-Brenkenhoff F, Bernhardt $\mathrm{P}$, et al. Clinical Characteristics and Cardiovascular Magnetic Resonance Findings in Stress (Takotsubo) Cardiomyopathy. JAMA 2011;306:277-86.

19. Ghadri JR, Wittstein I, Prasad A, et al. International Expert Consensus Document on Takotsubo Syndrome (Part I): Clinical Characteristics, Diagnostic Criteria, and Pathophysiology. Eur Heart J 2018;39:2032-46.

20. Ghadri JR, Wittstein I, Prasad A, et al. International Expert Consensus Document on Takotsubo Syndrome (Part II): Diagnostic Workup, Outcome, and Management. Eur Heart J 2018;39:2047-62. 